\title{
Irrational Use of Antibiotics Leading to No Bacterial Growth on Culture
}

\author{
Naveed Altaf ${ }^{1}$, Farhana Aljabri ${ }^{2}$, K.S. Saraswathi ${ }^{3}$ and R. Shyamala ${ }^{4 *}$ \\ ${ }^{1}$ Department of Pharmacology, ${ }^{2}$ Department of OBGY, \\ ${ }^{3}$ Department of Obstetrics and Gynaecology, Shadan Institute of Medical \\ Sciences and Research Centre, Hyderabad 500008, Telangana State, India \\ ${ }^{4}$ Department of Microbiology, Malla reddy Medical College for Women, \\ Hyderabad, Telangana State, India \\ *Corresponding author
}

\section{A B S T R A C T}

\begin{tabular}{|l|}
\hline Ke y w o r d s \\
UTI, No Bacterial \\
Growth, \\
Antibiotics, \\
Resistance
\end{tabular}

Urinary tract infection is an infection that affects part of the urinary tract. When it affects lower urinary tract it is known as cystitis, when it affects upper urinary tract it is known as pyelonephritis. Urinary tract infections occur more commonly in women than men. The objectives of this study is to analyse the urine samples from patients who complained of signs and symptoms of UTI. The Culture reports were analysed. Urine culture sensitivity reports were analysed for a period of one year from January 2016 to December 2016 and found that out of 248 samples, 186 samples (i.e, 75\%) had a report of no bacterial growth. In these patients there was indiscriminate use of several courses of antibiotics. The remaining samples showed Escherichia coli, Klebsiella species and Proteus species. The process of antibiotic resistance is increased when the antibiotics are underused, overused or misused. Before starting treatment for UTI, urine should be sent for culture \& sensitivity and the correct antibiotic therapy should be administered.

\section{Introduction}

Drug resistance refers to tolerance of microorganisms to inhibitory action of antimicrobials example staphylococci to penicillin.

Natural Resistance- Some microbes have always been resistant to certain AntiMicrobial agents. Example Gram-negative bacilli are normally unaffected by Penicillin $\mathrm{G}$, aerobic organisms are not affected by metronidazole, while anaerobic bacteria are not inhibited by aminoglycoside antibiotics, or Mycobacterium tuberculosis is insensitive to tetracycline. This type of resistance does not pose a significant clinical problem (Tripathi, 1998).

Acquired resistance is a major clinical problem. It is the development of by an organism which was sensitive before due to the use of an aminoglycoside over a period of time (Laxminarayan, 2003; Dromigny et al., 2003) this can happen with any microbe. This development of resistance is dependent on the microorganism as well as the drug. If antibiotic are used irrationally it leads to resistance to antibiotics (Zuccato et al., 2005) Data regarding culture sensitivity for 
antibiotics was analysed. Patients who came to our hospital with signs and symptoms of urinary tract infections was collected, data regarding culture sensitivity to antibiotics was analysed. It was found that the patients who used various antibiotics prior to coming to the hospital had no bacterial growth. This study was done for establishing antibiotic policy by pharmacovigilance cell of our hospital, where the patient gives history of taking several courses of antibiotics. Prior to coming to hospital, and at the same time the incorrect dosage of antibiotics - usually develops drug resistance; and the result of cultures shows no bacterial growth.

An effective antibiotic policy should be framed for the hospital and strict adherence to this, will lead to decrease in the irrational use of antibiotics.

\section{Materials and Methods}

Analysis of urine culture and sensitivity reports was done for a period of one year from January 2016 to December 2016. The urine samples on reaching the laboratory were inoculated on MacConkey agar, Blood agar and Nutrient agar to isolate the organisms. The inoculated Nutrient agar and Blood agar plates were incubated aerobically at 37 degree Celsius for 24 hours. After overnight incubation at 37 degree Celsius the Blood agar and MacConkey agar were examined for evidence of growth.

The colony characters were studied, smears were stained by Grams' stain and examined under the $100 \mathrm{X}$ objective. The bacterial species then isolated were identified by morphology, cultural characteristics and biochemical reactions according to the standard techniques.[Cruickshank R] The Gram negative bacilli identified were tested for motility by hanging drop and then were subjected to biochemical and sugar fermentation tests. The tests were read after incubation at 37 degree $\mathrm{C}$ at the end of $24 \mathrm{hrs}$ and 48 hrs. Antibiotic Sensitivity tests were done by Kirby Bauer disk diffusion method.

\section{Results and Discussion}

Urine culture sensitivity reports were analysed for a period of one year from January 2016 to December 2016 and found that out of 248 samples, 186 samples (i.e, $75 \%$ ) had a report of no bacterial growth which doesn't give any clue of the organism (Davies, 1990).

62 samples have shown positive culture report. Out of these 62 samples, 44 samples (17.74\%) showed Escherichia coli, 15 samples (6.04\%) showed Klebsiella species and 3 samples (1.22\%) showed Proteus species.

Antimicrobial chemotherapy has been a leading cause for the dramatic rise of average life expectancy in the Twentieth Century. Disease causing microbes have become resistant to antibiotic drug therapy and this is an increasing medical problem. For many years, antibiotics have been critical in the fight against infectious disease caused by bacteria and other microbes (Stephen $\mathrm{T}$. Odonkor et al., 2011).

This study was done as a part of devising an antibiotic policy by Pharmacovigilance cell at the tertiary care centre of Shadan hospital. Data regarding culture and sensitivity for antibiotics was analysed in patients who presented with clinical signs and symptoms of UTI. No bacterial growth was seen in patients who gave history of prior use of various antibiotics, or improper use of antibiotics. When antibiotics are underused, the process of antibiotic resistance is increased. 


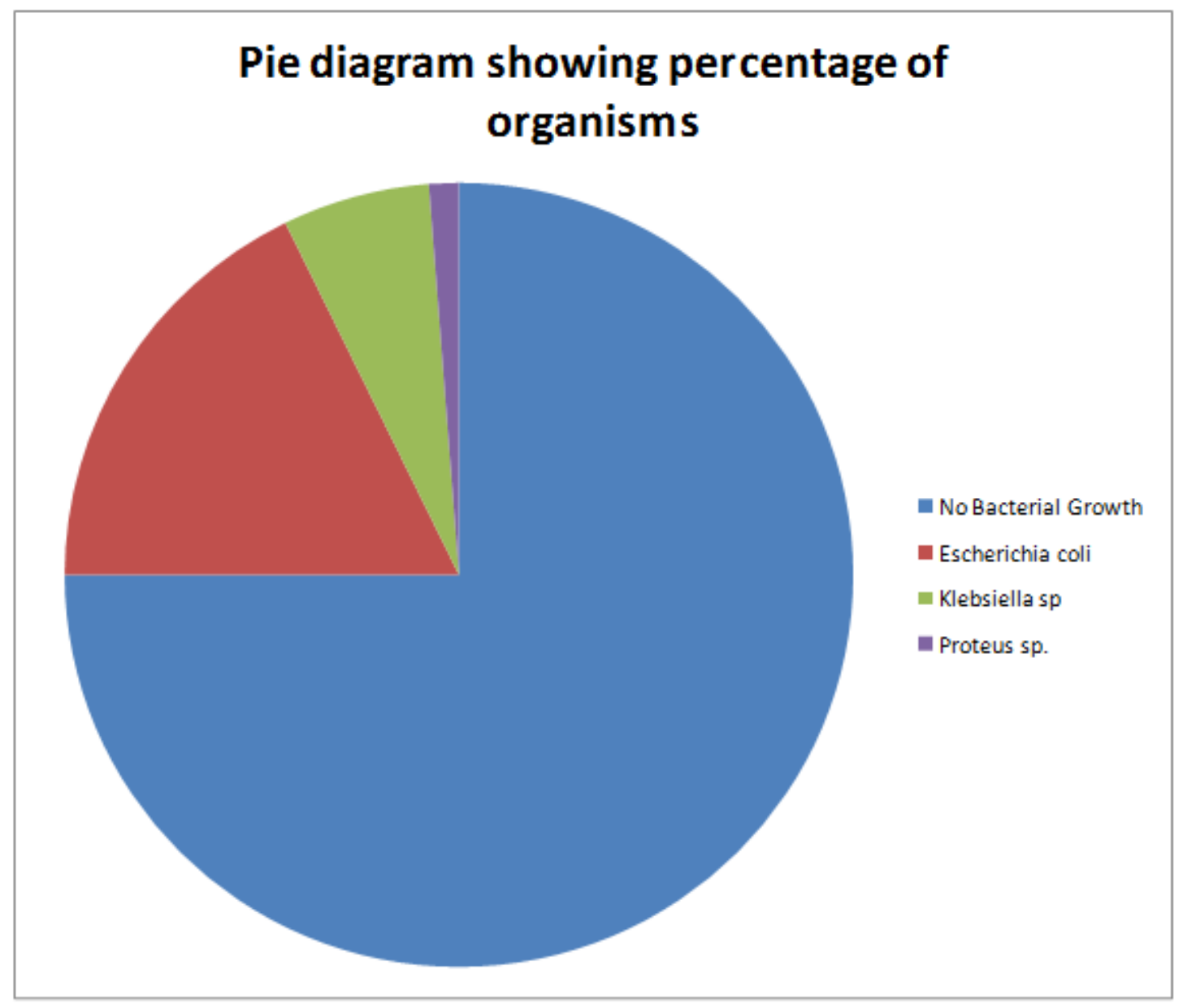

When the patients receive prescriptions for antibiotics, they should complete the full course of therapy to ensure that all pathogens die and should not save any pills for later use. Patients should refrain from demanding antibiotics for colds and other viral infections and might consider seeking antibiotic therapies for minor conditions.

Irrational use of antibiotics leads to No Bacterial Growths which further lead to increased usage of antibiotics with incorrect diagnosis. Hence use of antibiotics after lab reports with correct diagnosis will decrease adverse drug reactions and resistance to drugs and prevent no bacterial growths in culture sensitivity reports and thus yielding proper results (Deepalatha et al., 2011).
It is mandatory that urine samples should be sent for culture \& sensitivity in patients with Urinary Tract Infections and correct antibiotic to be given for correct duration to prevent complications and antibiotic resistance.

\section{References}

Cruickshank, R., Medical microbiology: The practice of Medical microbiology, Churchill Livingstone, U.K., 199, 3, 374

Davies, A.J., 1990 Journal of Antimicrobial Chemotherapy, 10: 7-9

Deepalatha, C., et al., Journal of Microbiology and Biotechnology Research, 2011, 1(4):1111-113

Dromigny, J.A., et al., Antimicrobial reistance of Salmonella enteric serotype 
Typhi in Dakar, Senegal. Clin Infect Dis. 2003; 37: 465-466

Laxminarayan, R., Battling resistance to antibiotics and pesticides: an economic approach. Washington, DC: Resources for the future. 2003.

Stephen T. Odonkor, et al., International Journal of Biological and Medical Research, 2011; 2(4): 1204-1210
Tripathi, K.D., Essentials of Medical Pharmacology, Seventh Edition, Jaypee publishers, 1998.pp. 69.

Zuccato, E., et al., Identification of the pharmaceuticals for human use contaminating the Italian aquatic environment. Journal of Hazardous Materials. 2005; 122(3), 205e209

\section{How to cite this article:}

Naveed Altaf, Farhana Aljabri, K.S. Saraswathi and Shyamala, R. 2017. Irrational Use of Antibiotics Leading to No Bacterial Growth on Culture. Int.J.Curr.Microbiol.App.Sci. 6(9): 1688-1691. doi: https://doi.org/10.20546/ijcmas.2017.609.207 